\title{
Spleen Preservation in Laparoscopic Distal Pancreatectomy for Solid Pseudopapillary Neoplasm is Oncologically Safe
}

\author{
Yongjoon Won, M.D., Yoo-Seok Yoon, M.D., Ph.D., Ho-Seong Han, M.D., Ph.D., Jai Young Cho, M.D., Ph.D., \\ YoungRok Choi, M.D., Ph.D., In Gun Hyun, M.D., Kil Hwan Kim, M.D. \\ Department of Surgery, Seoul National University Bundang Hospital, Seoul National University College of Medicine, Seongnam, Korea
}

Purpose: Laparoscopic distal pancreatectomy (LDP) has been widely performed for solid pseudopapillary neoplasm (SPN) involving the body or tail of the pancreas. However, it has not been established whether spleen preservation in LDP is oncologically safe for the treatment of SPN with malignant potential. In this study, we compared the short- and long-term outcomes between patients with SPN who underwent laparoscopic spleen-preserving distal pancreatectomy (LSPDP) vs laparoscopic distal pancreatectomy with splenectomy (LDPS).

Methods: We retrospectively reviewed the medical records of 46 patients with SPN who underwent LDP between January 2005 and November 2016. Patients were divided into 2 groups according to spleen preservation: the LSPDP group $(n=32)$ and the LDPS group $(n=14)$. Clinicopathologic characteristics and perioperative outcomes were compared between groups.

Results: There were no significant differences in pathologic variables, including tumor size, tumor location, node status, angiolymphatic invasion, or perineural invasion between groups. Median operating time was significantly longer in the LSPDP group vs the LDPS group (243 vs 172 minutes; $p=0.006$ ). Estimated intraoperative blood loss was also significantly greater in the LSPDP group (310 vs $167 \mathrm{ml} ; p=0.063)$. There were no significant differences in incidence of postoperative complications ( $\geq$ Clavien-Dindo class IIIa) or pancreatic fistula between groups. After a median follow-up of 35 months (range, 3 153 months), there was no recurrence or disease-specific mortality in either group.

Conclusion: The results show that LSPDP is an oncologically safe procedure for SPN involving the body or tail of the pancreas.

Keywords: Laparoscopic distal pancreatectomy, Spleen preservation, Solid pseudopapillary neoplasm

This is an Open Access article distributed under the terms of the Creative Commons Attribution Non-Commercial License (http:/ creativecommons.org/licenses/by-nc/4.0/) which permits unrestricted non-commercial use, distribution, and reproduction in any medium, provided the original work is properly cited.
Received July 3, 2018

Revised 1st August 12, 2018 2nd September 5, 2018

Accepted September 16, 2018

Corresponding author

Yoo-Seok Yoon

Department of Surgery, Seoul

National University Bundang

Hospital, 82 Gumi-ro 173beon-gil,

Bundang-gu, Seoungnam 13620,

Korea

Tel: +82-31-787-7099

Fax: +82-31-787-4055

E-mail: yoonys@snubh.org

ORCID:

http://orcid.org/0000-0001-7621-8557
Copyright (๑) 2019 The Journal of Minimally Invasive Surgery. All rights reserved.

\section{INTRODUCTION}

Solid pseudopapillary neoplasms (SPNs) are rare pancreatic cystic neoplasms with low malignant potential, accounting for approximately $1 \%$ to $2 \%$ of all pancreatic tumors. ${ }^{1}$ With the widespread use of radiologic imaging studies and increased recognition of this disease entity, the number of reported cases of SPN has been increasing. Surgical resection is the treatment of choice for localized SPNs, and the prognosis after complete resection is excellent, with a 5-year survival rate of $>95 \%$. ${ }^{2}$ Recently, laparoscopic distal pancreatectomy (LDP) has been widely performed for SPN involving the body or tail of the pancreas, and the spleen-preserving method is frequently used with advances in laparoscopic techniques., ${ }^{3,4}$ However, it 
has not been established whether spleen preservation in LDP is oncologically safe for the treatment of SPN with malignant potential. In this study, we compared the short- and long-term outcomes between patients with SPN who underwent laparoscopic spleen-preserving distal pancreatectomy (LSPDP) vs laparoscopic distal pancreatectomy with splenectomy (LDPS).

\section{MATERIALS AND METHODS}

\section{Patient and clinical variables}

A total of 46 patients with SPN who underwent LDP at Seoul National University Bundang Hospital between January 2005 and November 2016 were enrolled in this single-center, retrospective study. Patients were divided into 2 groups ac- cording to spleen preservation: the LSPDP group $(n=32)$ and the LDPS group ( $n=14)$. If technically feasible, LSPDP was performed except in cases where spleen vessel preservation is difficult due to the location of tumor in contact with splenic hilum and splenic vessels. The following data retrieved from a prospective database were analyzed to compare the clinical outcomes between groups: preoperative variables (age, sex, body mass index [BMI], initial symptoms, and preoperative tumor markers [carcinoembryonic antigen (CEA), carbohydrate antigen 19-9 (CA 19-9)]), pathologic data (tumor size, node status, number of lymph nodes harvested, and surgical margin status), and perioperative data (operating time, estimated blood loss, frequency and severity of postoperative complications based on the Clavien-Dindo system, incidence of pancreatic fistula according to the International Study

Table 1. Clinicopathologic characteristics

\begin{tabular}{|c|c|c|c|}
\hline & LDPS (n=14) & LSPDP (n=32) & $p$ value \\
\hline Sex & & & 0.010 \\
\hline Male & $7(50)$ & $4(12.5)$ & \\
\hline Female & $7(50)$ & $28(87.5)$ & \\
\hline Age, y & $43(18 \sim 78)$ & $38(11 \sim 72)$ & 0.314 \\
\hline $\mathrm{BMI}, \mathrm{kg} / \mathrm{m}^{2}$ & $24.1(20.0 \sim 28.7)$ & $23.9(17.6 \sim 43.8)$ & 0.783 \\
\hline Incidental detection & $8(57.1)$ & $12(37.5)$ & 0.333 \\
\hline Weight loss & $1(7.1)$ & $3(9.4)$ & 0.807 \\
\hline Abdominal pain & $3(21.4)$ & $9(28.1)$ & 0.729 \\
\hline Fever & $0(0)$ & $1(3.1)$ & 0.508 \\
\hline CEA, ng/ml & $1.4(0.0 \sim 4.2)$ & $0.5(0.0 \sim 2.4)$ & 0.098 \\
\hline CA 19-9, U/ml & $11.5(5.0 \sim 34.0)$ & $9.5(0.0 \sim 37.0)$ & 0.573 \\
\hline Tumor size, cm & $3.8(2.2 \sim 8.0)$ & $4.0(1.7 \sim 9.5)$ & 0.763 \\
\hline Tumor location & & & 0.316 \\
\hline Body & $3(21.4)$ & $13(40.6)$ & \\
\hline Tail & $11(78.6)$ & $19(59.4)$ & \\
\hline N stage & & & 0.212 \\
\hline $\mathrm{Nx}$ & $4(28.6)$ & $16(50)$ & \\
\hline NO & $10(71.4)$ & $16(50)$ & \\
\hline N1 & $0(0)$ & $0(0)$ & \\
\hline No. of LNs harvested & $5.8(2 \sim 12)$ & $3.3(1 \sim 11)$ & 0.082 \\
\hline Angiolymphatic invasion & $0(0)$ & $1|3.1\rangle$ & 0.508 \\
\hline Perineural invasion & $4(28.6)$ & $5(15.6)$ & 0.423 \\
\hline Surgical margin positive & $0(0)$ & $0(0)$ & \\
\hline
\end{tabular}

Data are presented as $n(\%)$ or median (range). BMI = body mass index; CA 19-9 = carbohydrate antigen 19-9; CEA = carcinoembryonic antigen; LDPS = laparoscopic distal pancreatectomy with splenectomy; LN = lymph node; LSPDP = laparoscopic spleen-preserving distal pancreatectomy. 
Group of Pancreatic Surgery definition, ${ }^{5}$ postoperative hospital stay, recurrence, and survival). This study was approved by the Institutional Review Board of Seoul National University Bundang Hospital.

\section{Surgical technique}

Detailed surgical techniques for LSPDP and LDPS have been described elsewhere. ${ }^{6,7}$ Briefly, under general anesthesia, the patient was placed in a $30^{\circ}$ reverse-Trendelenburg position with left side-up adjustment. After creation of pneumoperitoneum via an umbilical port, 2 trocars for the surgeon and 1 or 2 trocars for the assistant were inserted. Locations of the trocars varied according to the tumor location. The pancreas was approached after division of the greater omentum. The superior and inferior borders of the pancreas were dissected until the splenic artery and splenic vein were identified. After making a window between the pancreas and splenic vessels, the pancreas was transected using an endoscopic stapler. In the case of LSPDP, the pancreas was dissected from the splenic vessels in a medial-to-lateral fashion until the end of the pancreas. The small vessel branches encountered during dissection were controlled with metallic clips or energy devices. In the case of LDPS, the splenic artery and splenic vein were isolated and divided. The pancreas was then dissected from the retroperitoneum toward the spleen. With division of the short gastric vessels, splenectomy was performed. The surgical specimen was retrieved in a vinyl bag and extracted through a small incision created by extending a port-site incision. A Jackson-Pratt drain was placed near the pancreatic stump.

\section{Statistical analysis}

Continuous variables are presented as median (range). Continuous variables were compared using the nonparametric Mann-Whitney $U$ test, while categorical variables were compared using the Chi-square test or Fisher's exact test. A $p$ value of $<0.05$ was considered statistically significant. Statistical analysis was performed using SPSS version 18.0 (IBM Corporation, Armonk, NY).

\section{RESULTS}

\section{Clinicopathologic characteristics}

Clinicopathologic characteristics of the 2 groups are summarized in Table 1 . There were 11 men and 35 women with a median age of 39 years (range, 11 78 years). Among 46 patients, 20 had incidentally detected SPNs (LDPS group, 8/14 [57.1\%]; LSPDP group, 12/32 [37.5\%]). The most common symptom at the time of diagnosis was abdominal pain. There were more female patients in the LSPDP group. There were no significant differences in age, BMI, initial symptom, or preoperative tumor markers between groups.

Pathologically there were no significant differences in tumor size, tumor location, angiolymphatic invasion, or perineural

Table 2. Perioperative clinical and oncologic outcomes

\begin{tabular}{|c|c|c|c|}
\hline & LDPS (n=14) & $\operatorname{LSPDP}(n=32)$ & $p$ value \\
\hline Open conversion & $0(0)$ & $0(0)$ & \\
\hline Intraoperative transfusion & $0(0)$ & $1(3.1)$ & 0.508 \\
\hline Operating time, min & $172(95 \sim 325)$ & $243(95 \sim 555)$ & 0.006 \\
\hline Estimated blood loss, ml & $167(0 \sim 400)$ & $310(0 \sim 1500)$ & 0.063 \\
\hline Overall complications & & & 0.721 \\
\hline ( $\geq$ Grade Illa) & $1(7.1)$ & $5(15.6)$ & \\
\hline Portal vein thrombosis & $1(7.1)$ & $0(0)$ & 0.304 \\
\hline POPF & & & 0.739 \\
\hline Grade B & $1(7.1)$ & $1(3.1)$ & \\
\hline Grade C & $0(0)$ & $0(0)$ & \\
\hline Postoperative hospital stay, d & $9.7(7 \sim 19)$ & $10.3(5 \sim 45)$ & 0.721 \\
\hline Recurrence & $0(0)$ & $0(0)$ & \\
\hline Mortality & $0(0)$ & $0(0)$ & \\
\hline
\end{tabular}

Data presented as $n(\%)$ or median (range). LDPS = laparoscopic distal pancreatectomy with splenectomy; LSPDP = laparoscopic spleen-preserving distal pancreatectomy; POPF = postoperative pancreatic fistula. 
invasion between groups. Number of lymph nodes harvested tended to be higher in the LDPS group, but the difference between groups was not significant. No nodal metastasis was found in either group. Surgical resection margins were negative in all patients in both groups.

\section{Perioperative outcomes}

Intra- and postoperative outcomes of the 2 groups are summarized in Table 2. Conversion to open surgery was not required in either group. Intraoperative transfusion was required in 1 patient in the LSPDP group. Median operating time was significantly longer in the LSPDP group vs the LDPS group (243 vs 172 minutes; $p=0.006$ ). Estimated blood loss was also significantly greater in the LSPDP group (310 vs $167 \mathrm{ml}$; $p=0.063$ ). Of the 32 cases in the LSPDP group, we performed the Warshaw technique in 8 patients and splenic vessel preservation in 24 patients. In cases of splenic vessel preserving LSPDP, the splenic artery patency rate was 91.6\% (22/24) and the splenic vein patency rate was $66.6 \%$ (16/24). There were no significant differences in incidence of postoperative complications ( $\geq$ Clavien-Dindo class IIIa) or pancreatic fistula between groups. Postoperative hospital stay was similar in both groups. After a median follow-up of 35 months (range, 3 153 months), there was no recurrence or disease-specific mortality in either group.

\section{DISCUSSION}

The results of this study show that spleen preservation did not have a negative impact on short- or long-term outcomes of LDP for SPN. There were no significant differences in postoperative complications, including incidence of pancreatic fistula, between groups. In particular, there was no recurrence or disease-related mortality in either group. However, LSPDP was associated with longer operating time and greater intraoperative blood loss compared with LDPS, which may be inevitable because of the technical difficulty related to preservation of the splenic vessels.

SPNs have a relatively good prognosis if curative resection is performed, demonstrating a 5-year survival rate of $95 \%$ to $100 \%{ }^{2,9}$ The majority of SPNs are located in the pancreatic body or tail, requiring distal pancreatectomy. However, the optimal surgical extent for this disease entity is not well established. In the case of left-sided pancreatic cancer, distal pancreatectomy with splenectomy is typically recommended for oncologic resection due to frequent invasion to the splenic vessels and possible metastasis to the lymph nodes in the hilum of the spleen. However, it is questionable whether spleen resection is necessary for the treatment of SPNs with low malignant potential and rare incidence of lymph node metastasis. $^{10}$ In this study, metastasis to the lymph nodes was not observed in any patient, although lymph nodes were not retrieved in approximately $40 \%$ of patients (20 of 46). None of the patients experienced recurrence, irrespective of lymph node retrieval.

Previous studies have reported that most patients with SPNs who underwent curative resection did not experience recurrence. Types of recurrence include local recurrence, liver metastasis, and intra-abdominal dissemination. Most SPN relapses are systemic. ${ }^{11}$ What is important is whether R0 resection is achieved. Radical lymphatic dissection and splenectomy for local control do not seem to affect patient outcomes. ${ }^{10,12}$ Other reports comparing splenectomy and spleen preservation in LDP have also demonstrated that spleen preservation did not worsen survival outcomes. ${ }^{13-15}$

This study has several limitations, including its retrospective nature and small number of patients. Although not included in the data collection of this study, $\mathrm{Ki}-67$ was found to be positive in less than $1 \%$ in most cases. The short duration of median follow up is a limitation of this study and it should be reinforced by other studies. To definitely confirm the oncologic safety of spleen preservation in LDP for SPN, largescale, well-designed studies are necessary in the future.

In conclusion, LSPDP for SPN is a technically demanding procedure, requiring longer operating time and greater intraoperative blood loss compared with LDPS. However, this surgical procedure does not increase postoperative complications and does not decrease oncologic safety.

\section{REFERENCES}

1) Naar L, Spanomichou DA, Mastoraki A, Smyrniotis V, Arkadopoulos N. Solid Pseudopapillary Neoplasms of the Pancreas: A Surgical and Genetic Enigma. World J Surg 2017;41:1871-1881.

2) Papavramidis T, Papavramidis S. Solid pseudopapillary tumors of the pancreas: review of 718 patients reported in English literature. J Am Coll Surg 2005;200:965-972.

3) Afridi SA, Kazaryan AM, Marangos IP, et al. Laparoscopic surgery for solid pseudopapillary tumor of the pancreas. JSLS 2014;18: 236-242.

4) Jarry J, Bodin R, Peycru T, Nunez M, Collet D, Cunha AS. Role of laparoscopic distal pancreatectomy for solid pseudopapillary tumor. JSLS 2012;16:552-558.

5) Eshmuminov D, Schneider MA, Tschuor C, et al. Systematic review and meta-analysis of postoperative pancreatic fistula rates using the updated 2016 International Study Group Pancreatic Fistula definition in patients undergoing pancreatic resection with soft and hard pancreatic texture. HPB (Oxford) 2018 May 25 [Epub]. DOI: 10.1016/j.hpb.2018.04.003. 
6) Kim S, Yoon YS, Han HS, et al. A blunt dissection technique using the LigaSure vessel-sealing device improves perioperative outcomes and postoperative splenic-vessel patency after laparoscopic spleen- and splenic-vessel-preserving distal pancreatectomy. Surg Endosc 2018;32:2550-2558.

7) Yoon YS, Lee KH, Han HS, Cho JY, Ahn KS. Patency of splenic vessels after laparoscopic spleen and splenic vessel-preserving distal pancreatectomy. Br J Surg 2009;96:633-640.

8) Stark A, Donahue TR, Reber HA, Hines OJ. Pancreatic Cyst Disease: A Review. JAMA 2016;315:1882-1893.

9) de Castro SM, Singhal D, Aronson DC, et al. Management of solid-pseudopapillary neoplasms of the pancreas: a comparison with standard pancreatic neoplasms. World J Surg 2007;31:1130-1135.

10) Vassos N, Agaimy A, Klein P, Hohenberger W, Croner RS. Solidpseudopapillary neoplasm (SPN) of the pancreas: case series and literature review on an enigmatic entity. Int J Clin Exp Pathol 2013;6:1051-1059.

11) Kang CM, Choi SH, Kim SC, Lee WJ, Choi DW, Kim SW. Pre- dicting recurrence of pancreatic solid pseudopapillary tumors after surgical resection: a multicenter analysis in Korea. Ann Surg 2014; 260:348-355.

12) Yu P, Cheng X, Du Y, et al. Solid Pseudopapillary Neoplasms of the Pancreas: a 19-Year Multicenter Experience in China. J Gastrointest Surg 2015;19:1433-1440.

13) Nakamura $Y$, Matsushita A, Mizuguchi $Y$, Katsuno A, Uchida E. Study on laparoscopic spleen preserving distal pancreatectomy procedures comparing splenic vessel preservation and non-preservation. Transl Gastroenterol Hepatol 2016;1:27.

14) Uchida H, Goto C, Kishimoto H, et al. Laparoscopic spleen-preserving distal pancreatectomy for solid pseudopapillary tumor with conservation of splenic vessels in a child. J Pediatr Surg 2010;45: 1525-1529.

15) Melotti G, Butturini G, Piccoli M, et al. Laparoscopic distal pancreatectomy: results on a consecutive series of 58 patients. Ann Surg 2007;246:77-82. 